\begin{tabular}{|c|c|c|}
\hline & $\begin{array}{l}\text { European Association for the } \\
\text { Development of Renewable Energies, Environment } \\
\text { and Power Quality (EA4EPQ) }\end{array}$ & $\begin{array}{l}\text { International Conference on Renewable Energies and Power Quality } \\
\text { (ICREPQ'12) } \\
\text { Santiago de Compostela (Spain), } 28^{\text {th }} \text { to } 30^{\text {th }} \text { March, } 2012\end{array}$ \\
\hline
\end{tabular}

\title{
Parameter Identification of a PV Source Dynamic Model with Piecewise-Linear Circuit-Based Approach
}

\author{
M. C. Di Piazza ${ }^{1}$, Member IEEE, M. Luna ${ }^{1}$, G. Vitale ${ }^{1}$, Member IEEE \\ ${ }^{1}$ Consiglio Nazionale delle Ricerche, Istituto di Studi sui Sistemi Intelligenti \\ per l'Automazione (ISSIA - CNR), sezione di Palermo, Palermo, Italy. \\ mariacarmela.dipiazza@ieee.org; luna@pa.issia.cnr.it; gianpaolo.vitale@ieee.org
}

\begin{abstract}
This paper deals with a method for the parameter identification of an improved dynamic model of a photovoltaic (PV) source, which encompasses a capacitor and an inductor. The method is based on piecewise-linear (PWL) circuit approximation and step load responses. Considering the PV I-V characteristic as a PWL function, if a transient occurs in a linear region, the PV source can be approximated according to Thévenin's theorem. The cases referred to either capacitive or inductive dominant time constant are explained. The identification of the circuit parameters by least squares regression (LSR) applied to experimental data is discussed and finally a case study is presented.
\end{abstract}

Keywords- Photovoltaic source; dynamic modeling; statistics; least squares regression.

\section{INTRODUCTION}

An accurate mathematical model of the PV source represents a useful tool for the analysis of power converters in PV plants, for the study of maximum power point tracking (MPPT) algorithms and, above all, for simulating the PV system using circuit simulators or real time emulators. Several papers dealing with static PV modeling have been published. In general, diode-based nonlinear models are used, mostly a double-diode configuration [1-2] or a single-diode model [3-7].

However, either for theoretical investigations or for emulating purposes, a dynamic model is often necessary. because the connected load cannot be considered constant.

In order to model the electrical dynamics of a PV source, a variable or fixed capacitor is usually added to the static model [8-9]. However, the resulting first order circuit does not allow to reproduce second order behaviors caused by the interaction of the junction capacitance with the parasitic inductance, mainly introduced by cables and metallic connections. Such typical behaviors are resonances on DC cables, interaction with inverter switching frequency harmonics and underdamped currents due to load variation.

Hence, besides the capacitor, an inductor has to be added to the components of any static PV model (single-diode, double-diode or others), to improve the dynamic model of the PV source. As an example, fig. 1 shows the dynamic model obtained starting from the single-diode model.

The proposed dynamic parameter identification is based on the consideration that the PV I-V characteristic can be considered as a piecewise linear (PWL) function [10-11]. As a consequence, if a transient involving a linear part of the characteristic occurs, the source can be modeled as a voltage generator with a series resistance, according to Thévenin's theorem. A suitable choice of the load resistor ensures noninteracting capacitor and inductor time constants, allowing the parameters $C$ and $L$ to be identified. The situation $\tau_{C}>10 \cdot \tau_{L}$ represents a reduced number of $\mathrm{PV}$ modules with short cables. On the contrary, a large number of PV modules with long cables implies $\tau_{L}>10 \cdot \tau_{C}$. Both scenarios represent typical cases.

The paper is organized as follows: after discussing steady-state and transient analyses, a method for the identification of the circuit parameters is explained; finally a case study is presented and conclusions are given.

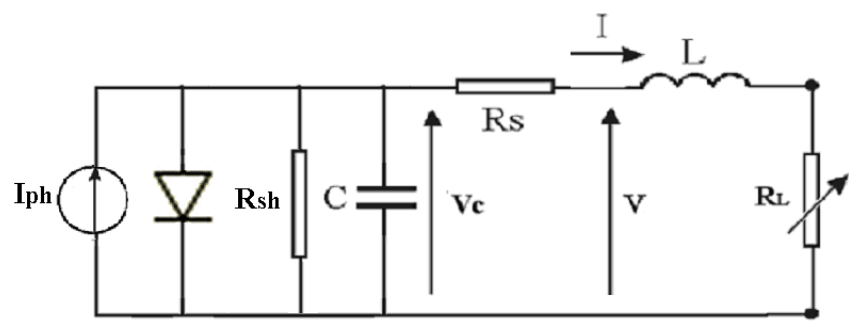

Fig. 1. Improved dynamic model of the PV source based on single-diode static model

\section{STEADY-STATE ANALYSIS}

The equivalent circuit of a PV source in a linear piece of its I-V characteristic is drawn in fig. 2. It can be noted that, compared to the single-diode model, the current source with parallel diode and the shunt resistor have been replaced with a voltage generator $E_{t h}$ with a series resistance $R_{t h}$. This linear circuit is different from the mere series or parallel RLC circuit, so a specific analysis is necessary.

The approach proposed in this paper consists in the application of a step load variation to the considered circuit

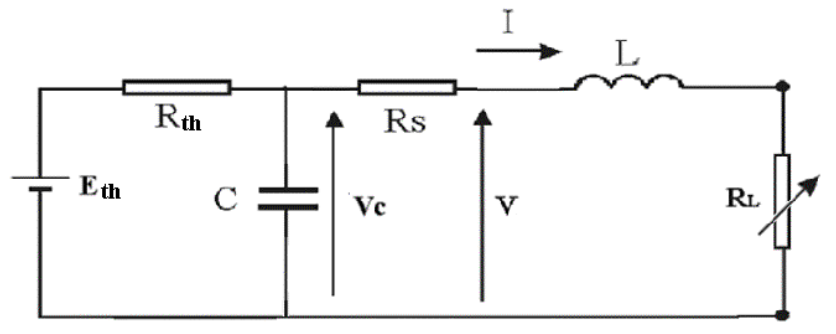

Fig. 2. The considered RLC circuit which is equivalent to a PV source operating in a linear zone. 
in steady-state; the old value is indicated by $\mathrm{R}_{\mathrm{L} \text {,old }}$, while then new one is simply denoted by $\mathrm{R}_{\mathrm{L}}$. In general, the time instants immediately before and after the load variation are indicated with $\mathrm{t}=0^{-}$and $\mathrm{t}=0^{+}$, respectively; the time at which all transients have expired is denoted with the infinity symbol.

In order to determine the response of this circuit it is worth noting that at $\mathrm{t}=0^{-}$it is:

$$
\left\{\begin{array}{l}
I\left(0^{-}\right)=\frac{E_{\text {th }}}{R_{\text {th }}+R_{L, \text { old }}+R_{s}} \stackrel{\Delta}{=} I_{\text {old }} \\
V\left(0^{-}\right)=R_{L, \text { old }} \cdot I\left(0^{-}\right)=\frac{E_{\text {th }} \cdot R_{L, \text { old }}}{R_{\text {th }}+R_{L, \text { old }}+R_{s}} \stackrel{\Delta}{=} V_{\text {old }} \\
V_{C}\left(0^{-}\right)=\frac{E_{t h} \cdot\left(R_{L, \text { old }}+R_{s}\right)}{R_{\text {th }}+R_{L, \text { old }}+R_{s}}=V_{\text {old }}+R_{s} I_{\text {old }} \stackrel{\Delta}{=} V_{C, \text { old }}
\end{array}\right.
$$

At $\mathrm{t}=0^{+}$, due to the presence of the inductor and the capacitor, no variation occurs, so it is:

$$
\left\{\begin{array}{c}
I\left(0^{+}\right)=I\left(0^{-}\right)=I_{\text {old }} \\
V\left(0^{+}\right)=V\left(0^{-}\right)=V_{\text {old }} \\
V_{C}\left(0^{+}\right)=V_{C}\left(0^{-}\right)=V_{C, \text { old }}
\end{array}\right.
$$

Instead, at the steady-state following the load variation it is:

$$
\left\{\begin{array}{l}
I(\infty)=\frac{E_{t h}}{R_{t h}+R_{L}+R_{s}} \stackrel{\Delta}{=} I_{\infty} \\
V(\infty)=R_{L} \cdot I(\infty)=\frac{E_{t h} \cdot R_{L}}{R_{t h}+R_{L}+R_{s}} \stackrel{\Delta}{=} V_{\infty} \\
V_{C}(\infty)=\frac{E_{t h} \cdot\left(R_{L}+R_{s}\right)}{R_{t h}+R_{L}+R_{s}}
\end{array}\right.
$$

\section{TRANSIENT ANALYSIS WITH $\tau_{C}>10 \cdot \tau_{L}$}

When the capacitive effect is dominant, the time constant $\tau_{C}$ tied to the capacitance is much greater than the time constant $\tau_{L}$ tied to the inductance. In this case, after the step load variation, firstly the inductor drives the transient, remaining the capacitor at a nearly constant voltage. Then, when the inductor's energy remains constant, it behaves as a short circuit and the capacitor begins charging or discharging. These two phases, indicated as inductive and capacitive transient respectively, can be analysed separately and the related quantities will be denoted with subscripts ind and cap.

A typical response of the circuit in fig. 2 with $\tau_{C}>10 \cdot \tau_{L}$ is shown in fig.s $3 \mathrm{a}-3 \mathrm{~b}$ for e.g. $\mathrm{R}_{\mathrm{L}}<\mathrm{R}_{\mathrm{L} \text {,old }}$, together with the capacitive and inductive transients.

As the figures show, the initial conditions of the inductive transient equal the corresponding conditions of the circuit in fig. 2. Instead, the steady-state conditions of the inductive transient equal the initial conditions of the capacitive transient. Furthermore, the steady-state conditions of the capacitive transient equal the corresponding conditions of the circuit in fig. 2.

The analysis of the inductive and capacitive transients with $\tau_{C}>10 \cdot \tau_{L}$ is presented in Appendix A. For the sake of simplicity, only the main results are recalled here.

As for the inductive transient, its time constant is given by:

$$
\tau_{L}=L /\left(R_{L}+R_{s}\right)
$$

and its steady-state values are expressed by:

$$
\left\{\begin{array}{l}
I_{\text {ind }}(\infty)=\frac{V_{C, \text { old }}}{R_{s}+R_{L}} \stackrel{\Delta}{=} I_{0} \\
V_{\text {ind }}(\infty)=\frac{V_{C, \text { old }} \cdot R_{L}}{R_{s}+R_{L}} \stackrel{\Delta}{=} V_{0}
\end{array}\right.
$$

Then, current $I_{\text {ind }}$ is given by:

$$
\begin{aligned}
& I_{\text {ind }}(t)=\left[I_{\text {ind }}(\infty)-I_{\text {ind }}\left(0^{+}\right)\right] \cdot\left(1-e^{-t / \tau_{L}}\right)+I_{\text {ind }}\left(0^{+}\right)= \\
& =\left[I_{0}-I_{\text {old }}\right] \cdot\left(1-e^{-t / \tau_{L}}\right)+I_{\text {old }}
\end{aligned}
$$

Plotting (6) produces the related green curve of fig. $3 b$, which is a close approximation of the response of the circuit in fig. 2 before the effect of the capacitor starts to be significant.

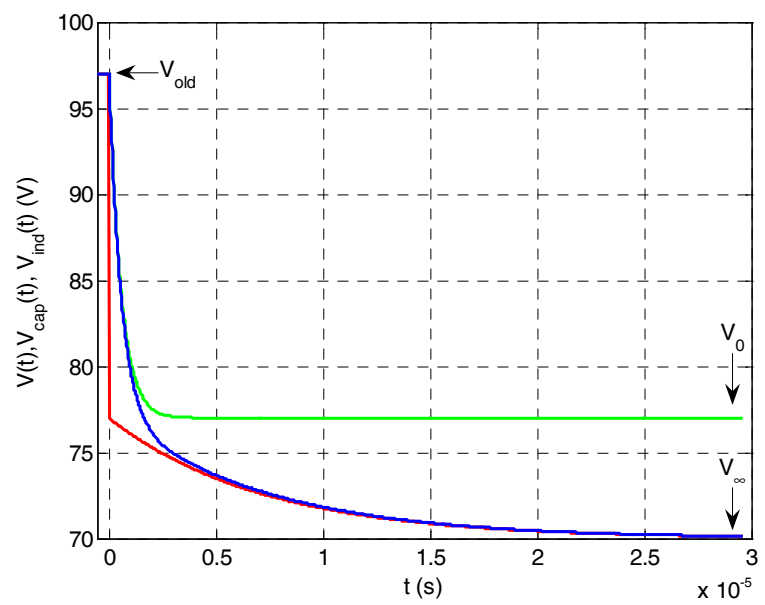

Fig. 3a. Voltage $\mathrm{V}$ (blue curve), voltage $\mathrm{V}_{\text {cap }}$ (red) and voltage $\mathrm{V}_{\text {ind }}$ (green) for $\tau_{\mathrm{C}}>10 \cdot \tau_{\mathrm{L}}$

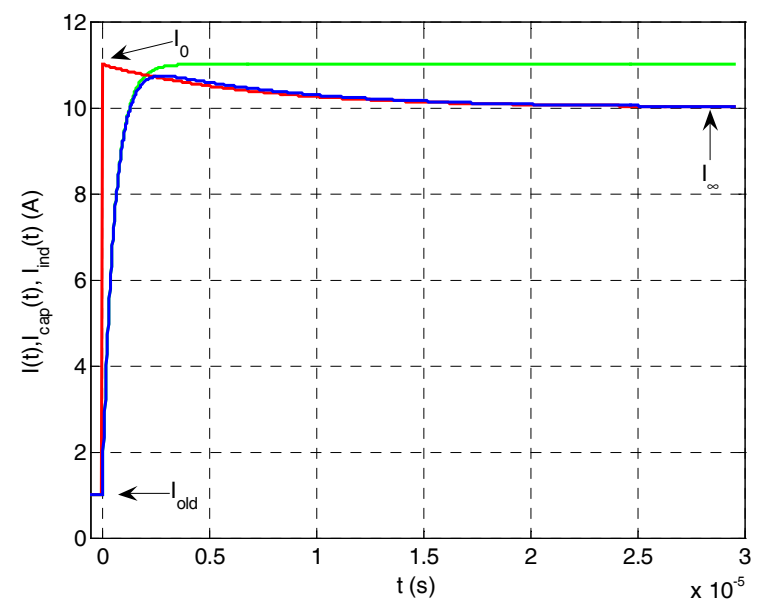

Fig. 3b. Current I (blue curve), current $\mathrm{I}_{\text {cap }}$ (red) and current $\mathrm{I}_{\text {ind }}$ (green) for $\tau_{\mathrm{C}}>10 \cdot \tau_{\mathrm{L}}$

As for the capacitive transient, its time constant is given by:

$$
\tau_{C}=R_{e q} \cdot C
$$

with:

$$
R_{e q}=\frac{R_{t h}\left(R_{L}+R_{s}\right)}{R_{t h}+R_{L}+R_{s}}
$$


Then, current $I_{\text {cap }}$ is expressed by:

$$
\begin{aligned}
I_{c a p}(t) & =\left[I_{c a p}\left(0^{+}\right)-I_{c a p}(\infty)\right] \cdot e^{-t / \tau_{c}}+I_{c a p}(\infty)= \\
& =\left[I_{0}-I_{\infty}\right] \cdot e^{-t / \tau_{c}}+I_{\infty}
\end{aligned}
$$

Plotting (9) produces the related red curve of fig. 3b, which is a close approximation of the response of the circuit in fig. 2 after that the effect of the inductor has expired.

Now it is possible to observe that, just as for any RLC circuit, if the chosen time origin equals the instant the step load variation is applied, the load current of the circuit in fig. 2 is given by the following equation:

$$
I(t)=-A_{1} \cdot e^{-t / \tau_{1}}+A_{2} \cdot e^{-t / \tau_{2}}+I_{\infty}
$$

It is possible to rearrange (10) in the following form:

$$
I(t)=I_{1}(t)+I_{2}(t)-K
$$

where:

$$
\left\{\begin{aligned}
I_{1}(t) & =A_{1} \cdot\left(1-e^{-t / \tau_{1}}\right)+I_{\text {old }} \\
I_{2}(t) & =A_{2} \cdot e^{-t / \tau_{2}}+I_{\infty} \\
K & =A_{1}+I_{\text {old }}
\end{aligned}\right.
$$

Comparing (12a),(12b) with (6) and (9) respectively, it is possible to deduce that:

$$
\left\{\begin{array}{l}
I_{\text {ind }}(t)=I_{1}(t) \text { with } A_{1}=I_{0}-I_{\text {old }} \text { and } \tau_{1}=\tau_{L} \\
I_{\text {cap }}(t)=I_{2}(t) \text { with } A_{2}=I_{0}-I_{\infty} \text { and } \tau_{2}=\tau_{C}
\end{array}\right.
$$

This confirms that when the capacitive effect is dominant, the circuit's response can be obtained summing the inductive and capacitive transients, which can be analysed separately, and then shifting down the obtained waveform by $\mathrm{K}$ according to (11). Therefore a transient with $\tau_{C}>10 \cdot \tau_{L}$ can be exploited to determine the values of $C$ and $L$.

\section{TRANSIENT ANALYSIS WITH $\tau_{L}>10 \cdot \tau_{C}$}

In this case, after the step load variation, firstly the capacitor drives the transient, remaining nearly constant the inductor current. Then, when the capacitor's energy remains constant, it behaves as an open circuit and the inductor begins charging or discharging.

Now the initial conditions of the capacitive transient equal the corresponding conditions of the circuit in fig. 2; instead, the steady-state conditions of the capacitive transient equal the initial conditions of the inductive transient.

Furthermore, the steady-state conditions of the inductive transient equal the corresponding conditions of the circuit in fig. 2.

The analysis of the capacitive and inductive transients with $\tau_{L}>10 \cdot \tau_{C}$ is presented in Appendix B. For the sake of simplicity, only the main results are recalled here.

Because of the specific initial conditions, the capacitive transient collapses into one point so the response of the real circuit shown in fig. 2 is identical to the inductive transient:

$$
I_{\text {ind }}(t)=\left[I_{\infty}-I_{\text {old }}\right] \cdot\left(1-e^{-t / \tau_{L 2}}\right)+I_{\text {old }}
$$

with:

$$
\tau_{L 2}=L /\left(R_{L}+R_{t h}+R_{s}\right)
$$

This means that the analysis of a transient with $\tau_{L}>10 \cdot \tau_{C}$ cannot be exploited to determine the value of $C$; it allows to determine only the value of $L$.

\section{PARAMETER IDENTIFICATION}

The equations presented in section III allow the identification of the circuit parameters through a least squares regression (LSR) applied to experimental data obtained during a reference test, during which the load current waveform is acquired while applying a step variation of a purely resistive load.

The reference test must be performed with a load resistance such that $\tau_{C}>10 \cdot \tau_{L}$, so that an overshoot appears in the waveform. This is possible since both time constants depend on $\mathrm{R}_{\mathrm{L}}$, which can be chosen suitably.

\section{A. Transition from $R_{L, \text { old }} \neq \infty$ to $R_{L} \neq R_{L, \text { old }}$}

From the acquired waveform it is possible to read the following quantities: $V_{\text {old }}, I_{\text {old }}, V_{\infty}$ and $I_{\infty}$. In particular, if $R_{L, o l d}=0$, it is $V_{\text {old }}=0$ and $I_{\text {old }}$ equals the short circuit current, so the equations which follow can be simplified.

As a preliminar step, in order to determine $\mathrm{E}_{\mathrm{th}}$ it is possible to combine (1a) and (3a), obtaining:

$$
E_{\text {th }}=\frac{R_{L}-R_{L, \text { old }}}{1 / I_{\infty}-1 / I_{\text {old }}}
$$

Looking at the current's waveform $I(t)$, if the peak time is referred to as $t^{*}$ and $\Delta T_{1}, \Delta T_{2}$ correspond to suitable time intervals, it is possible to observe that:

$$
\left\{\begin{array}{l}
\left.I(t)\right|_{0} ^{\Delta T_{1}}=\left.I_{\text {ind }}(t)\right|_{0} ^{\Delta T_{1}} \\
\left.I(t)\right|_{t^{+}+\varepsilon} ^{t^{*}+\varepsilon+\Delta T_{2}}=\left.I_{\text {cap }}(t)\right|_{t^{t}+\varepsilon} ^{t^{t}+\varepsilon+\Delta T_{2}}
\end{array}\right.
$$

where $\varepsilon$ is a suitable nonzero quantity.

In order to identify the capacitance value it is possible to combine (9) and (13b) obtaining:

$$
\ln \left(I_{\text {cap }}(t)-I_{\infty}\right)=\ln A_{2}-t / \tau_{C}=q_{1}+m_{1} \cdot t
$$

and, thus, to apply a LSR to the quantity:

$$
\mathrm{y}_{\mathrm{C}}=\ln \left[\left.I(t)\right|_{t^{t+\varepsilon}} ^{t^{+}+\varepsilon+\Delta T_{2}}-I_{\infty}\right]
$$

The opposite of the slope $m_{l}$ will give the inverse of the capacitive time constant $\tau_{C}$. The quantity $e^{q_{1}}$ equals $A_{2}$ and allows to determine $I_{0}$ according to (13b):

$$
I_{0}=e^{q_{1}}+I_{\infty}
$$

Then, combining (1c) and (5a) it is:

$$
R_{s}=\frac{R_{L} I_{0}-R_{L, \text { old }} I_{\text {old }}}{I_{\text {old }}-I_{0}}
$$

Furthermore, from (3a) or (1a) respectively, it is:

$$
R_{t h}=\frac{E_{t h}}{I_{\infty}}-R_{s}-R_{L} \text { or } R_{t h}=\frac{E_{t h}}{I_{\text {old }}}-R_{s}-R_{\text {Lold }}
$$

Now it is possible to determine $\mathrm{R}_{\mathrm{eq}}$ according to (8). 
Finally, the capacitance is given by:

$$
C=\tau_{C} / R_{e q}
$$

Similarly, in order to identify the inductance value, it is possible to combine (6) and (13a) obtaining:

$$
\ln \left(I_{0}-I_{\text {ind }}(t)\right)=\ln A_{1}-t / \tau_{L}=q_{2}+m_{2} \cdot t
$$

and, thus, to apply a LSR to the quantity:

$$
\mathrm{y}_{\mathrm{L}}=\ln \left[I_{0}-\left.I(t)\right|_{0} ^{\Delta T_{1}}\right]
$$

The opposite of the slope $m_{2}$ will give the inverse of the inductive time constant $\tau_{L}$, while the inductance will be given by:

$$
L=\tau_{L} \cdot\left(R_{L}+R_{s}\right)
$$

Finally, from (13a) it is $I_{0}=A_{1}+I_{\text {old }}=e^{q_{2}}+I_{\text {old }}$ so a new value of $I_{0}$ is obtained, which can be used to verify the goodness of the whole procedure, since it should equal the value of $I_{0}$ calculated with (20).

\section{B. Transition from $R_{L, \text { old }}=\infty$ to $R_{L} \neq R_{L, \text { old }}$}

When $R_{L, o l d}=\infty$ some specific considerations apply.

Firstly, it is $V_{\text {old }}=V_{C, \text { old }}=E_{\text {th }}$ and $I_{\text {old }}=0$. This implies that (16) is not needed.

Equation (21) reduces to an undetermined form. In this case it is possible to find an expression for $R_{s}$ from (5a):

$$
R_{s}=\frac{E_{t h}}{I_{0}}-R_{L}
$$

After the identification of the inductance value, the new value of $I_{0}$ can be calculated simply as $I_{0}=A_{1}=e^{q_{2}}$.

\section{EXAMPLE OF PARAMETER IDENTIFICATION}

This section provides an example of parameter identification applied to a PV generator with the following reference values:

$$
V_{o c}=19.58 \mathrm{~V}, I_{s c}=961.7 \mathrm{~mA}, V_{m p}=15 \mathrm{~V}, I_{m p}=894.8 \mathrm{~mA}
$$

The I-V characteristic of the PV array is shown in fig. 4 and it can be approximated with a 4-segment piecewiselinear characteristic. In particular, the so-called nearly constant current (I) and nearly constant voltage (IV) regions can be considered.

Then, a reference test can be performed with a load resistor value such that the operating point belongs to either of the afore mentioned regions. In these scenarios, the behaviour of the PV array can be assumed to be linear and described by the circuit of fig. 2 .

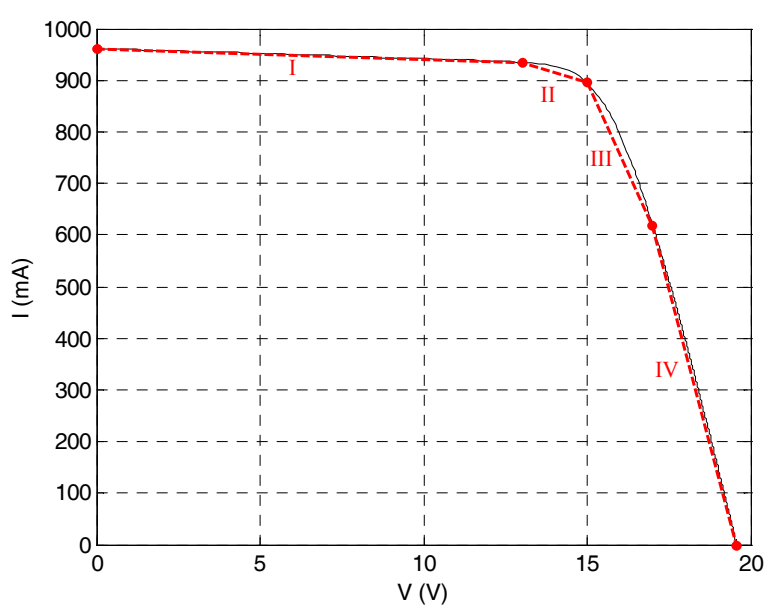

Fig. 4. PV characteristic (black) and its 4-segment PWL approximation (red)

\section{A. Transient on the nearly constant voltage region}

This reference test allows to determine the following parameters: $C, L$ and $R_{s}$ while $E_{t h}$ is taken from the I-V characteristic.

A transient is considered from the open circuit point $\left(R_{L, o l d}=\infty\right)$ to the midpoint of the curvilinear arc MPP-V $V_{O C}$ $\left(R_{L}=30 \Omega\right)$. The related current waveform has been acquired and plotted in fig. 5 (blue curve). For the sake of clarity, fig. 5 shows only a portion of the curve; the waveform actually starts at $I(0)=I_{\text {old }}=0$, ending at $I(\infty)=I_{\infty}=579.3 \mathrm{~mA}$.

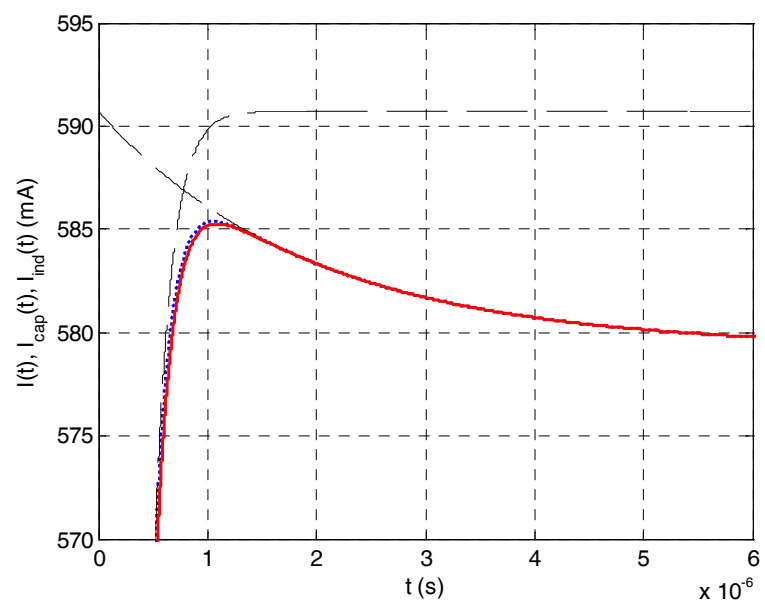

Fig. 5. Load current (blue, dotted), curves $\mathrm{I}_{\text {cap }}$ and $\mathrm{I}_{\text {ind }}$ (black, dashed) and their sum (red, solid) shifted by $K$.

Then, the PV source can be described by the set of components $\left(E_{t h}=V_{o c} ; R_{t h}=R_{s 0}-R_{s}\right)$, where $-1 / R_{s 0}$ is the slope of the nearly constant region [12]. However, in order to apply the procedure described in section V.B, only the knowledge of the Thévenin generator is required:

$$
E_{t h}=V_{o c}=19.58 \mathrm{~V}
$$

The peak time $\left(t^{*}=1.1 \cdot 10^{-6} \mathrm{~s}\right)$ and the steady-state load current $\left(I_{\infty}=579.3 \mathrm{~mA}\right)$ have been read on the graph of fig. 5. Then, after plotting (19) extended to the whole time interval (fig. 6), the chosen interval for the first LSR has been specified by means of the following parameters: $\varepsilon=4 \cdot 10^{-7} \mathrm{~s}, \Delta T_{2}=2 \cdot 10^{-6} \mathrm{~s}$. The regression line $y=q_{1}+m_{1} \cdot t$ has been superimposed. 
The opposite of the inverse of the slope has given the following capacitive time constant:

$$
\tau_{C}=1.929 \mu \mathrm{s}
$$

Subsequent calculations, allowed the following quantities to be determined:

$$
\begin{aligned}
& I_{0}=591 \mathrm{~mA} \\
& R_{s}=3.15 \Omega, R_{t h}=0.65 \Omega, R_{e q}=0.64 \Omega
\end{aligned}
$$

$(32 \mathrm{a}, \mathrm{b}, \mathrm{c})$

Then, the capacitance value has been determined according to $(23)$ :

$$
C=3.00 \mu \mathrm{F}
$$

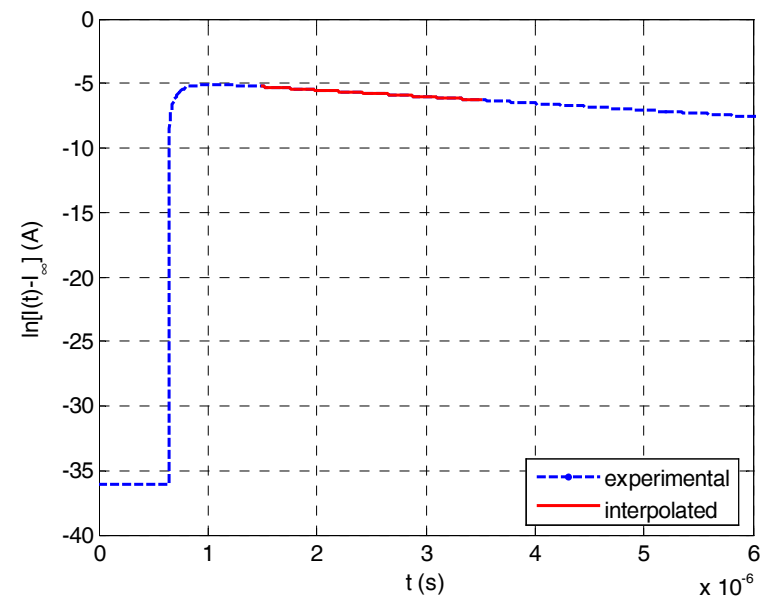

Fig. 6. Plot of $\ln \left[\mathrm{I}(\mathrm{t})-\mathrm{I}_{\infty}\right]$ vs. time, with the interpolating line superimposed.

In order to perform the second LSR, equation (25) extended to the whole time interval has been plotted (fig. 7) and the parameter $\Delta T_{I}=5 \cdot 10^{-7} \mathrm{~s}$ has been chosen. The regression line $y=q_{2}+m_{2} \cdot t$ has been superimposed.

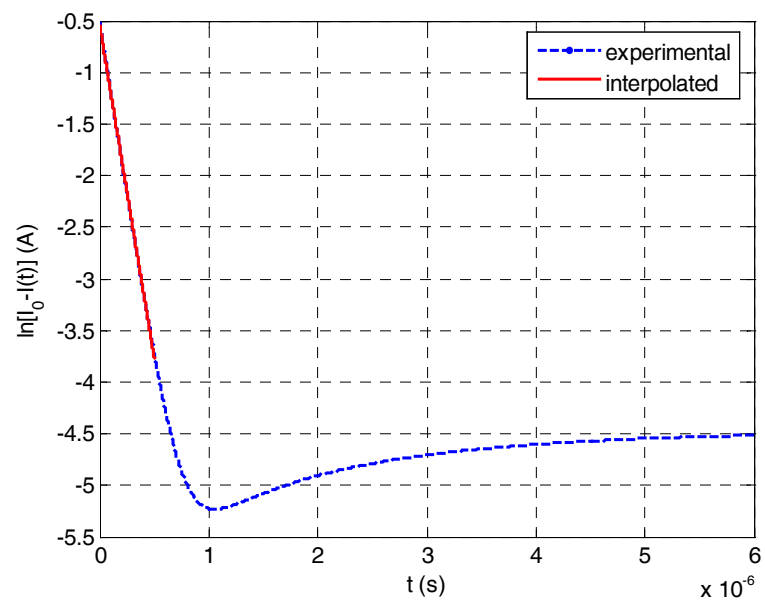

Fig. 7. Plot of $\ln \left[\mathrm{I}_{0}-\mathrm{I}(\mathrm{t})\right]$ vs. time, with the interpolating line superimposed.

The opposite of the inverse of the slope has given the following inductive time constant:

$$
\tau_{L}=155.1 \mathrm{~ns}
$$

Therefore, according to (26), the inductance value is:

$$
L=5.14 \mu \mathrm{H}
$$

In order to verify the goodness of the whole procedure, the quantity $e^{q_{2}}$ has been evaluated and compared to (31). A $1.8 \%$ error has been calculated, which is acceptable.

Finally, the curves $I_{\text {cap }}$ and $I_{\text {ind }}$ on which the load current waveform lies (black) and their sum (red) shifted down by $K$ have been plotted in fig. 5 to allow a comparison with the load current waveform (blue).

\section{B. Transient on the nearly constant current region}

This reference test allows to determine the following parameters: $C, L, R_{s h}$ and $I_{p h}$.

A transient is considered from the short-circuit point $\left(R_{L, \text { old }}=0 \Omega\right.$ ) to the midpoint of the curvilinear arc $I_{s c}-M P P$ $\left(R_{L}=10 \Omega\right)$. The related current waveform has been acquired and it is shown in fig. 8 (blue curve).

Then, in order to apply the procedure described in section V.A, the PV source can be described by the following set of components, whose values are unknown:

$\mathrm{E}_{\mathrm{th}}=\mathrm{I}_{\mathrm{ph}} \cdot \mathrm{R}_{\mathrm{sh}} ; \mathrm{R}_{\mathrm{th}}=\mathrm{R}_{\mathrm{sh}}$

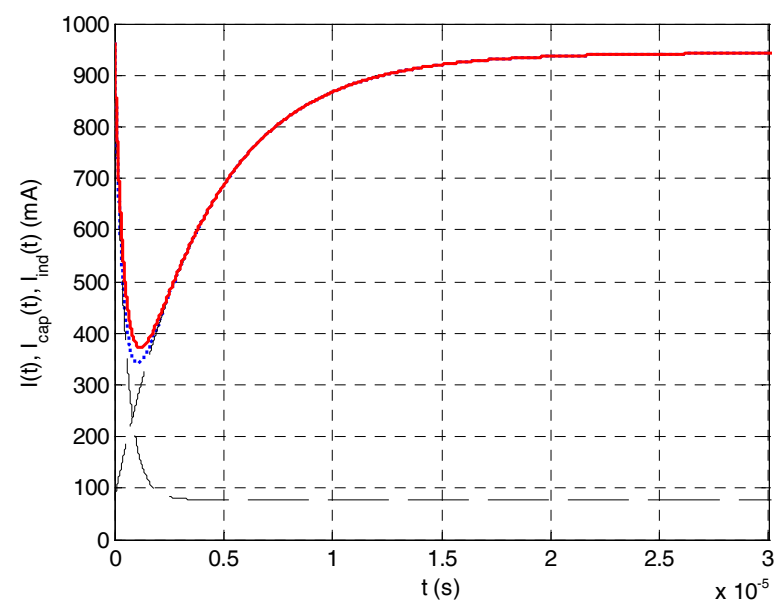

Fig. 8. Load current (blue, dotted), curves $\mathrm{I}_{\text {cap }}$ and $\mathrm{I}_{\text {ind }}$ (black, dashed) and their sum (red, solid) shifted by $K$.

The peak time $\left(t^{*}=1.07 \cdot 10^{-6} \mathrm{~s}\right)$ and the steady-state load current $\left(I_{\infty}=943 \mathrm{~mA}\right)$ have been read on the graph of fig. 8 . Then, the generator's value has been calculated according to (16):

$\mathrm{E}_{\mathrm{th}}=484 \mathrm{~V}$

After plotting (19) extended to the whole time interval (fig. 9), the chosen interval for the first LSR has been specified by means of the following parameters: $\varepsilon=9.3 \cdot 10^{-7} \mathrm{~s}, \Delta T_{2}=1.8 \cdot 10^{-5} \mathrm{~s}$. The regression line $y=q_{1}+m_{1} \cdot t$ has been superimposed.

The opposite of the inverse of the slope has given the following capacitive time constant:

$$
\tau_{C}=4.113 \mu \mathrm{s}
$$

Subsequent calculations allowed the following quantities to be determined:

$$
\begin{aligned}
& I_{0}=78.0 \mathrm{~mA} \\
& R_{t h}=502 \Omega, R_{e q}=10.6 \Omega
\end{aligned}
$$

Then, the capacitance value has been determined according to $(23)$ :

$$
C=386 \mathrm{nF}
$$


This value is different from (33). This is correct, since the junction capacitance is voltage-dependent and the operating point has changed.

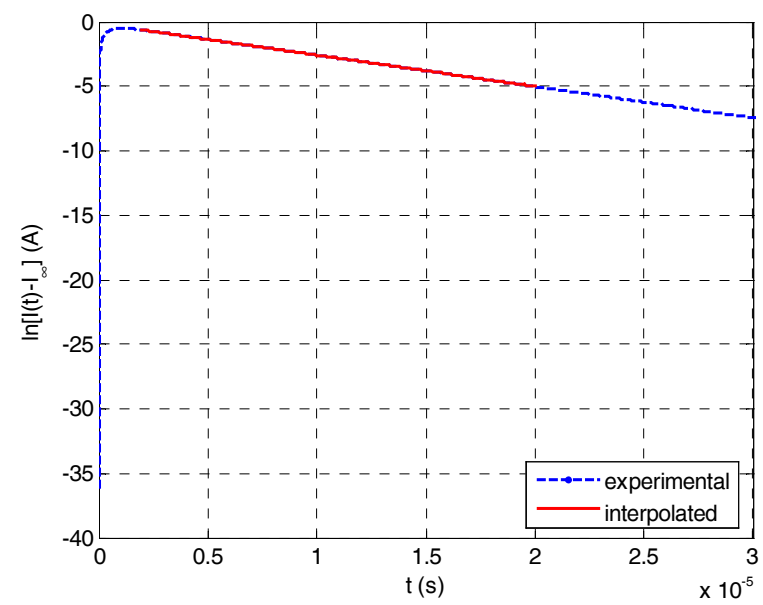

Fig. 9. Plot of $\ln \left[\mathrm{I}(\mathrm{t})-\mathrm{I}_{\infty}\right]$ vs. time, with the interpolating line superimposed.

In order to perform the second LSR, equation (25) extended to the whole time interval has been plotted (fig. 10) and the parameter $\Delta T_{l}=2 \cdot 10^{-7} \mathrm{~s}$ has been chosen. The regression line $y=q_{2}+m_{2} \cdot t$ has been superimposed.

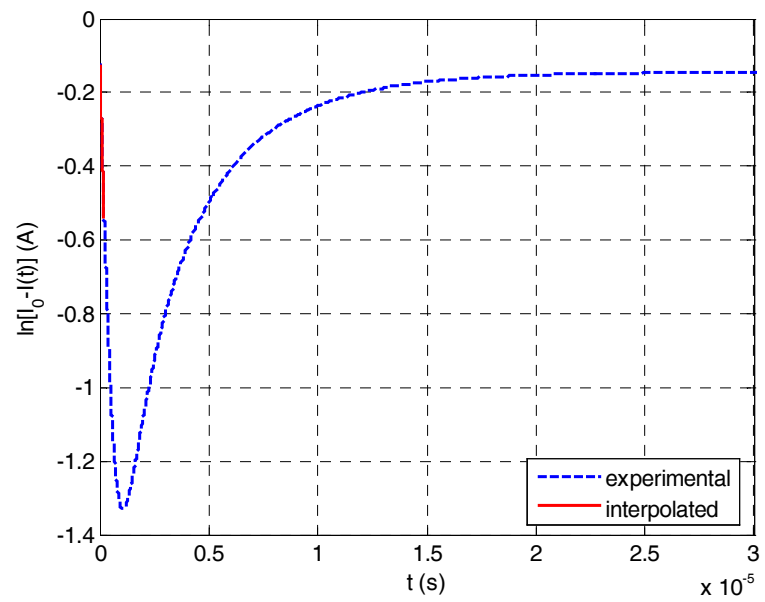

Fig. 10. Plot of $\ln \left[\mathrm{I}_{2}\left(0^{+}\right)-\mathrm{I}_{\text {load }}\right]$ vs. time, with the interpolating line superimposed.

The opposite of the inverse of the slope has given the following inductive time constant:

$$
\tau_{L}=487.1 \mathrm{~ns}
$$

Therefore, according to (26), the inductance value is:

$$
L=5.30 \mu \mathrm{H}
$$

Using the quantity $e^{q_{2}}$, a $-3.8 \%$ error against (39) has been calculated, which is acceptable.

As before, the curves $I_{\text {cap }}$ and $I_{\text {ind }}$ on which the load current waveform lies (black) and their sum (red) shifted down by $K$ have been plotted in fig. 8 to allow a comparison with the load current waveform (blue).

Finally, the unknown values have been determined:

$$
R_{s h}=R_{t h}=502.4 \Omega
$$

$$
I_{p h}=\frac{E_{t h}}{R_{t h}}=\frac{484}{502.4}=963.4 \mathrm{~mA}
$$

\section{CONCLUSIONS}

A method for the parameter identification of the dynamic model of a photovoltaic source, based on piecewise-linear circuit approximation and step load responses, has been studied. The model encompasses capacitive junction effects and parasitic inductive effects, which allow to reproduce second order behaviors.

The proposed approach is based on two reference tests, during which a step load variation is applied, obtaining an overshoot in the response waveform. The load values are chosen in order to perform transients involving the so-called nearly constant voltage and nearly constant current regions.

The first transient allows to determine the parameters $C$, $L$, and $R_{s}$ starting from the knowledge of $\mathrm{V}_{\mathrm{oc}}$. The other transient allows to determine $\mathrm{R}_{\mathrm{sh}}, \mathrm{I}_{\mathrm{ph}}$ and a different value of $\mathrm{C}$ for the new operating point.

In general, the proposed approach can be used also for studying other nonlinear circuits operating in linear regions.

\section{REFERENCES}

[1] Gow J. A. and Manning C. D. Development of a photovoltaic array model for use in power-electronics simulation studies. IEE Proc. Elect. Power Appl., vol. 146, no. 2, 1999, pp. 193-200.

[2] Hyvarinen J. and Karila J. New analysis method for crystalline silicon cells. Proc. 3rd World Conf. Photovoltaic Energy Convers., 2003, vol. 2, pp. 1521-1524.

[3] Chowdhury S., Taylor G. A., Chowdhury S. P., Saha A. K., and Song Y. H.. Modelling, simulation and performance analysis of a PV array in an embedded environment. Proc. 42nd Int. Univ. Power Eng. Conf. (UPEC), 2007, pp. 781-785.

[4] Pongratananukul N. and Kasparis T. Tool for automated simulation of solar arrays using general-purpose simulators. Proc. IEEE Workshop Comput. Power Electron., 2004, pp. 10-14.

[5] Di Piazza M. C., Luna M., Ragusa A., Vitale G. A dynamic model of a photovoltaic generator based on experimental data. Renewable Energy \& Power Quality Journal (RE\&PQJ), ISSN: 2172-038X , n. 8, April 2010. http://www.icrepq.com/rev-papers.htm

[6] Carrero C., Amador J., and Arnaltes S. A single procedure for helping PV designers to select silicon PV module and evaluate the loss resistances. Renewable Energy, 32 (15) (2007) 2579-2589.

[7] Di Piazza M.C., Vitale G. Photovoltaic field emulation including dynamic and partial shadow conditions. Applied Energy, 87; 2010 pp. 814-823.

[8] Chayavanich T., Limsakul C., Chayavanich N., Chenvidhya D., Jivacate C., Kirtikara K. Voltage and frequency dependent model for PV module dynamic impedance. Proc. 17th International Photovoltaic Science \& Engineering Conference, 2007.

[9] Gil-Arias O., Ortiz-Rivera E. I. A general purpose tool for simulating the behavior of PV solar cells, modules and arrays. Control and Modeling for Power Electronics. COMPEL 2008. 11th Workshop on 17-20 Aug. 2008, Page(s):1 - 5.

[10] Campbell R. C. A circuit-based photovoltaic array model for power system studies. Power Symposium, 2007. NAPS '07. 39th North American, Sept. 30 2007-Oct. 22007 Page(s):97 - 101.

[11] Xiao W., Dunford W.G., Capel A. A novel modeling method for photovoltaic cells. Proc. IEEE 35th Annual Power Electron. Spec. Conf., Aachen, Germany, 2004, pp.1950 - 1956.

[12] D. S. H. Chan, J. C. H. Fang. Analytical methods for the extraction of solar-cell single- and double-diode model parameters from I-V characteristics. IEEE Trans. on Electron. Devices, Vol. ED-34, No. 2 , February 1987. 
APPENDIX A

TRANSIENT ANALYSIS WITH $\tau_{C}>10 \cdot \tau_{L}$

\section{A. Inductive transient}

The capacitor $C$ shown in fig. 2 can be substituted with a voltage generator whose value equals $\mathrm{V}_{\mathrm{C} \text {,old }}$, obtaining the circuit in fig. 11. Then, the inductive time constant is given by:

$$
\tau_{L}=L /\left(R_{L}+R_{s}\right)
$$

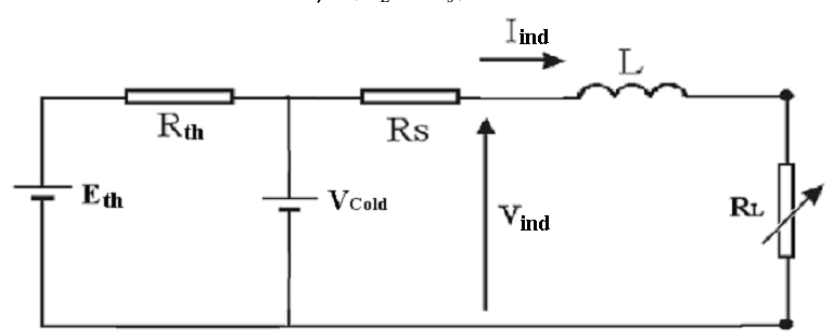

Fig. 11. Simplified circuit for studying the inductive transient when $\tau_{\mathrm{C}}>10 \cdot \tau_{\mathrm{L}}$

The initial conditions of the circuit in fig. 11 at $\mathrm{t}=0^{-}$and $\mathrm{t}=0^{+}$ are equal to those found for the circuit in fig. 2: $(1 a, b)$ and $(2 \mathrm{a}, \mathrm{b})$, respectively. However, its steady-state values, which will be the initial conditions of the capacitive transient, are different:

$$
\left\{\begin{array}{l}
I_{\text {ind }}(\infty)=\frac{V_{C, \text { old }}}{R_{s}+R_{L}} \stackrel{\Delta}{=} I_{0} \\
V_{\text {ind }}(\infty)=\frac{V_{C, \text { old }} \cdot R_{L}}{R_{s}+R_{L}} \stackrel{\Delta}{=} V_{0}
\end{array}\right.
$$

If, without loss of generality, a reduction of load resistance is supposed, then voltage $V_{\text {ind }}$ will start from its initial value (2b) and decrease with an exponential curve to reach its steady-state value expressed by (47b), according to fig. 3a:

$$
\begin{aligned}
& V_{\text {ind }}(t)=\left[V_{\text {ind }}\left(0^{+}\right)-V_{\text {ind }}(\infty)\right] \cdot e^{-t / \tau_{L}}+V_{\text {ind }}(\infty)= \\
& =\left[V_{\text {old }}-V_{0}\right] \cdot e^{-t / \tau_{L}}+V_{0}
\end{aligned}
$$

Similarly, according to fig. $3 \mathrm{~b}$, current $I_{\text {ind }}$ will be expressed by:

$$
\begin{aligned}
& I_{\text {ind }}(t)=\left[I_{\text {ind }}(\infty)-I_{\text {ind }}\left(0^{+}\right)\right] \cdot\left(1-e^{-t / \tau_{L}}\right)+I_{\text {ind }}\left(0^{+}\right)= \\
& =\left[I_{0}-I_{\text {old }}\right] \cdot\left(1-e^{-t / \tau_{L}}\right)+I_{\text {old }}
\end{aligned}
$$

In order to highlight the relationship between $V_{\text {ind }}(t)$ and $I_{\text {ind }}(t)$, it is possible to exploit (1c), obtaining:

$$
V_{\text {ind }}(t)=V_{C, \text { old }}-R_{s} I_{\text {ind }}(t)=V_{\text {old }}+R_{s} \cdot I_{\text {old }}-R_{s} \cdot I_{\text {ind }}(t)
$$

\section{B. Capacitive transient}

In this case, the inductor $\mathrm{L}$ in the circuit of fig. 2 can be substituted with a short-circuit obtaining the circuit in fig. 12. Then, the capacitive time constant is given by:

$$
\tau_{C}=R_{e q} \cdot C
$$

with:

$$
R_{e q}=\frac{R_{t h}\left(R_{L}+R_{s}\right)}{R_{t h}+R_{L}+R_{s}}
$$

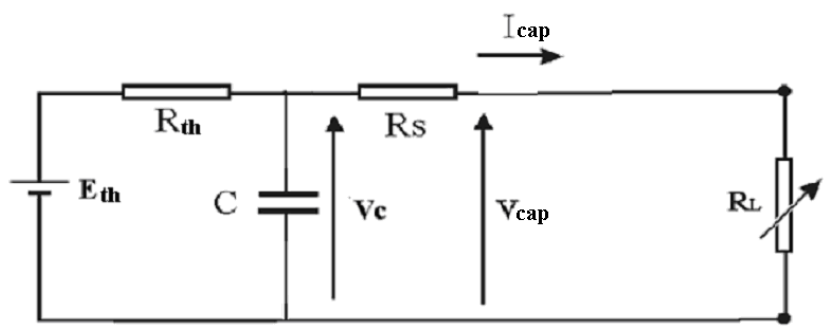

Fig. 12. Simplified circuit for studying the capacitive transient when $\tau_{\mathrm{C}}>10 \cdot \tau_{\mathrm{L}}$

The initial conditions of this circuit at $t=0^{-}$are equal to those found for the circuit in fig. 2, expressed by $(1 a, b, c)$. However, the values at $t=0^{+}$are different, since only the capacitor voltage stays constant now, while the other two quantities exhibit a discontinuity:

$$
\left\{\begin{array}{l}
I_{\text {cap }}\left(0^{+}\right)=\frac{V_{C}\left(0^{+}\right)}{R_{s}+R_{L}}=I_{0} \\
V_{\text {cap }}\left(0^{+}\right)=\frac{V_{C}\left(0^{+}\right) \cdot R_{L}}{R_{s}+R_{L}}=V_{0} \\
V_{C}\left(0^{+}\right)=V_{C}\left(0^{-}\right)=V_{C, \text { old }}
\end{array}\right.
$$

Furthermore, the circuit reaches the same steady-state values of the original RLC circuit in fig. 2 , i.e. (3a,b,c).

Observing the circuit of fig. 12 it is clear that $V_{\text {cap }}=R_{L} \cdot I_{\text {cap }}$, so, according to fig.s $3 \mathrm{a}-3 \mathrm{~b}$, voltage $V_{\text {cap }}$ and current $I_{\text {cap }}$ have the same trend, being expressed by:

$$
\begin{aligned}
V_{c a p}(t) & =\left[V_{c a p}\left(0^{+}\right)-V_{c a p}(\infty)\right] \cdot e^{-t / \tau_{c}}+V_{c a p}(\infty)= \\
& =\left[V_{0}-V_{\infty}\right] \cdot e^{-t / \tau_{c}}+V_{\infty} \\
I_{c a p}(t) & =\left[I_{c a p}\left(0^{+}\right)-I_{c a p}(\infty)\right] \cdot e^{-t / \tau_{c}}+I_{c a p}(\infty)= \\
& =\left[I_{0}-I_{\infty}\right] \cdot e^{-t / \tau_{c}}+I_{\infty}
\end{aligned}
$$

\section{APPENDIX B}

TRANSIENT ANALYSIS WITH $\tau_{L}>10 \cdot \tau_{C}$

A typical response of the circuit in fig. 2 with $\tau_{L}>10 \cdot \tau_{C}$ is shown in fig.s $13 \mathrm{a}-13 \mathrm{~b}$ for e.g. $R_{L}<R_{\text {Lold }}$ together with the capacitive and inductive transients.

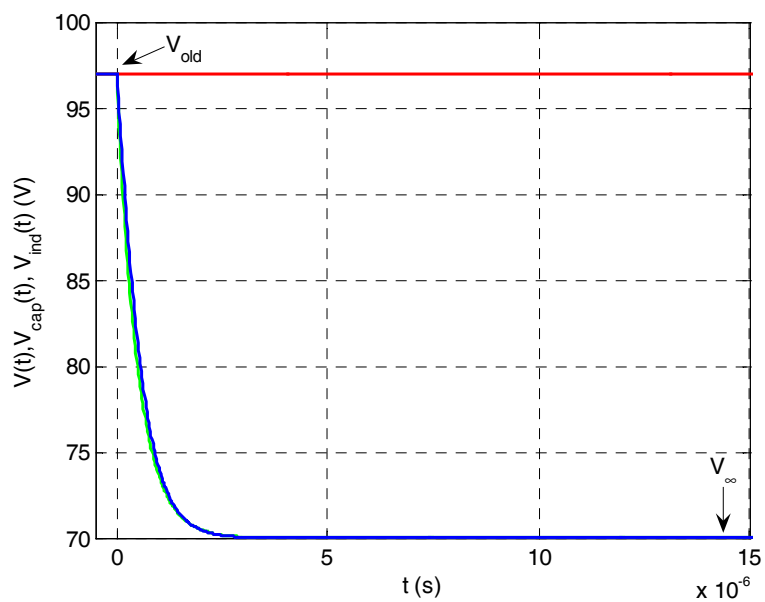

Fig. 13a. Voltage $V$ (blue curve), voltage $V_{\text {cap }}$ (red) and voltage $\mathrm{V}_{\text {ind }}$ (green) for $\tau_{\mathrm{L}}>10 \cdot \tau_{\mathrm{C}}$ 


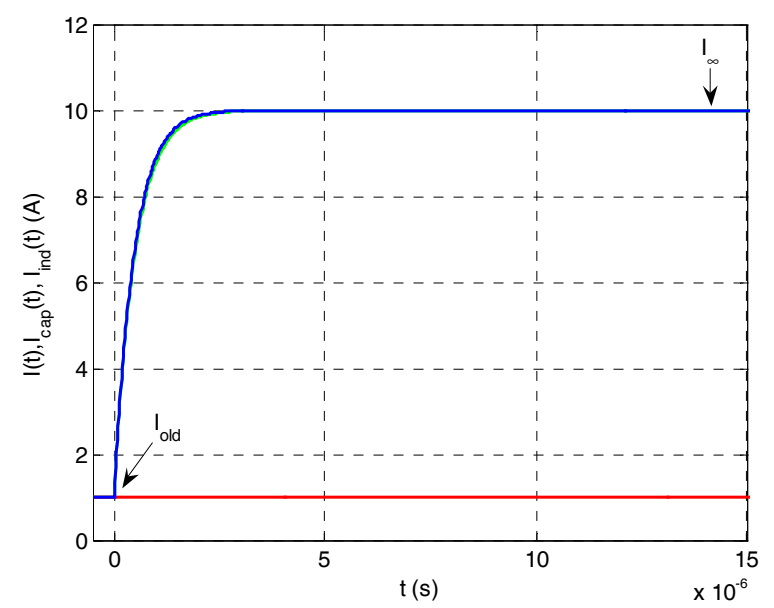

Fig. 13b. Current I (blue curve), current $\mathrm{I}_{\text {cap }}$ (red) and current $\mathrm{I}_{\text {ind }}$ (green) for $\tau_{\mathrm{L}}>10 \cdot \tau_{\mathrm{C}}$

\section{A. Capacitive transient}

The inductor $\mathrm{L}$ in the circuit of fig. 2 can be substituted with a current generator whose value equals $\mathrm{I}_{\text {old }}$, obtaining the circuit in fig. 14. Then, the new capacitive time constant is given by:

$$
\tau_{C 2}=R_{t h} \cdot C
$$

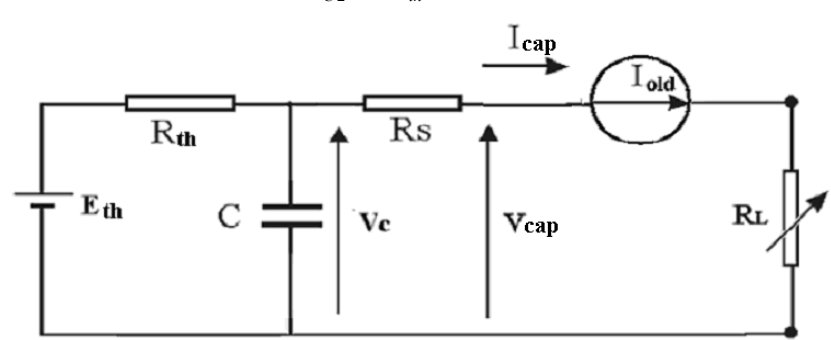

Fig. 14. Simplified circuit for studying the capacitive transient when $\tau_{\mathrm{L}}>10 \cdot \tau_{\mathrm{C}}$

The initial conditions of this circuit at $\mathrm{t}=0^{-}$and $\mathrm{t}=0^{+}$are equal to those found for the circuit in fig. 2 : $(1 \mathrm{a}, \mathrm{b}, \mathrm{c})$ and $(2 \mathrm{a}, \mathrm{b}, \mathrm{c})$, respectively. However, its steady-state values are different:

$$
\left\{\begin{array}{l}
I_{\text {cap }}(\infty)=I_{\text {old }} \\
V_{\text {cap }}(\infty)=V_{\text {old }} \\
V_{C}(\infty)=V_{C, \text { old }}
\end{array}\right.
$$

Therefore, given those specific initial conditions, this circuit exhibits no transient, so it is:

$$
\left\{\begin{array}{l}
I_{\text {cap }}(t)=I_{\text {old }} \\
V_{\text {cap }}(t)=V_{\text {old }}
\end{array}\right.
$$

As a matter of fact, assuming $\tau_{L}>10 \cdot \tau_{C}$ the inductor effects are immediately significant, so the capacitive approximation of the real response collapses into one point.

\section{B. Inductive transient}

The capacitor $C$ in the circuit of fig. 2 can be substituted with an open circuit, obtaining the circuit in fig. 15 . Then, the new inductive time constant is given by:

$$
\tau_{L 2}=L /\left(R_{L}+R_{t h}+R_{s}\right)
$$

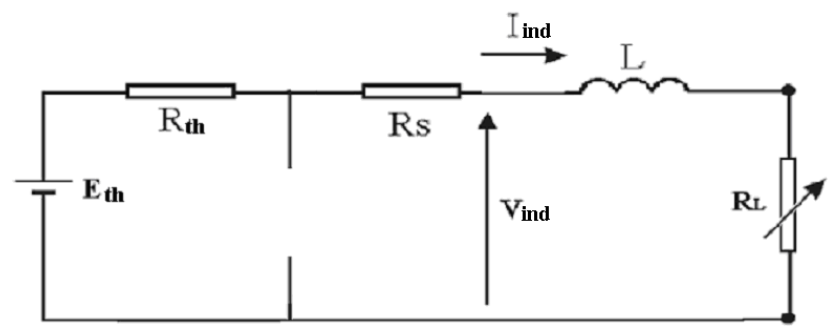

Fig. 15. Simplified circuit for studying the inductive transient when $\tau_{\mathrm{L}}>10 \cdot \tau_{\mathrm{C}}$

The initial conditions of this circuit at $\mathrm{t}=0^{-}$are equal to those found for the circuit in fig. 2, expressed by (1a,b). As for the conditions at $\mathrm{t}=0^{+}$, they are numerically equal to those found for the circuit in fig. 2 and to $(57 \mathrm{a}, \mathrm{b})$, as expected. Furthermore, the circuit reaches the same steadystate values of the original RLC circuit in fig. 2, described by equations $(3 a, b)$.

With similar considerations like those made in Appendix A it is possible to obtain:

$$
\begin{aligned}
I_{\text {ind }}(t) & =\left[I_{\infty}-I_{\text {old }}\right] \cdot\left(1-e^{-t / \tau_{L 2}}\right)+I_{\text {old }} \\
V_{\text {ind }}(t) & =\left[V_{\text {old }}-V_{\infty}\right] \cdot e^{-t / \tau_{L 2}}+V_{\infty}
\end{aligned}
$$

In order to highlight the relationship between $V_{\text {ind }}(t)$ and $I_{\text {ind }}(t)$, a quick look at the circuit in fig. 15 allows to deduce that:

$$
V_{\text {ind }}(t)=E_{t h}-\left(R_{t h}+R_{s}\right) \cdot I_{\text {ind }}(t)
$$

As previously said, the contribution of the capacitor is almost null so the response of the real circuit shown in fig. 2 is identical to the response of the simplified circuit in fig. 15 .

Furthermore, it is worth remarking that, when $0.1 \cdot \tau_{L} \leq \tau_{C} \leq 10 \cdot \tau_{L}$, the response of the circuit in fig. 15 is still a better approximation of the real circuit's response, compared to those of circuits in fig.s 11,12 and 14 . 